\title{
Bibliometric study of 'overviews of systematic reviews' of health interventions: evaluation of prevalence, citation and impact factor
}

Carole Lunny ( $\square$ carole.lunny@ubc.ca )

University of British Columbia https://orcid.org/0000-0002-7825-6765

\section{Trish Neelakant}

Royal College of Surgeons in Ireland

Alyssa Chen

University of British Columbia

Gavindeep Shinger

University of British Columbia

\section{Adrienne Stevens}

Michael G. DeGroote Cochrane Canada Centre https://orcid.org/0000-0002-6257-4806

\section{Sara Tasnim}

University of British Columbia

\section{Shadi Sadeghipouya}

University of British Columbia

\section{Stephen Adams}

University of British Columbia

\section{Yi Wen Zheng}

University of British Columbia

\section{Lester Lin}

University of British Columbia

\section{Pei Hsuan Yang}

University of British Columbia

\section{Manpreet Dosanjh}

University of British Columbia

\section{Peter Ngsee}

University of British Columbia

\section{Ursula Ellis}

University of British Columbia https://orcid.org/0000-0002-5896-4852

\section{Beverley J. Shea}

University of Ottawa https://orcid.org/0000-0002-7686-2585

\section{Emma K. Reid}


Nova Scotia Health

James M. Wright

University of British Columbia

\section{Method Article}

Keywords: overviews of reviews, overview of systematic reviews, umbrella reviews, meta-reviews, 'reviews of re-views', systematic reviews of systematic reviews, bibliometric, epidemiological characteristics, citation analy-sis, impact factor

Posted Date: June 28th, 2021

DOI: https://doi.org/10.21203/rs.3.rs-237881/v2

License: (c) (1) This work is licensed under a Creative Commons Attribution 4.0 International License. Read Full License 


\section{Abstract}

Background: Overviews synthesising the results of multiple systematic reviews help inform evidencebased clinical practice. In this first of two companion papers, we evaluate the bibliometrics of overviews, including their prevalence and factors affecting citation rates and journal impact factor.

Methods: We searched MEDLINE, Epistemonikos and Cochrane databases. We included overviews that: (a) synthesised reviews, (b) conducted a systematic search, (c) had a methods section, and (d) examined a healthcare intervention. Multivariate regression was conducted to determine the association between citation density, impact factor and 6 predictor variables.

Results: We found 1218 overviews published from 2000 to 2020; the majority (73\%) were published in the most recent 5-year period. We extracted a selection of these overviews ( $n=541 ; 44 \%)$ dated from 2000 to 2018. The 541 overviews were published in 307 journals; Cochrane Database (8\%), PLOS ONE (3\%) and Sao Paulo Medical Journal (2\%) were the most prevalent. The majority (70\%) were published in journals with impact factors between 0.05 and 3.97. We found a mean citation count of 10 overviews per year, published in journals with a mean impact factor of 4.4. In multivariate analysis, overviews with a high number of citations and impact factors had more authors, larger sample sizes, were open access and reported the funding source.

Conclusions: An 8-fold increase in the number of overviews was found between 2009 and 2020. We identified 332 overviews published in 2020, which is equivalent to 1 overview published per day. Overviews perform above average for the journals in which they publish.

\section{Background}

Bibliometric analysis measures the impact of articles using metrics such as the journal impact factor and number of citations [1]. A bibliometric analysis can serve to identify influential articles that have shaped medical practice, policy decisions, and presented new research ideas [2]. The journal impact factor measures the average number of citations of an article in the journal over the previous year. Citation analysis provides an objective measurement of how often scientists use specific published work. Although citations have limitations [3], they may offer vital information on how often scientific data are used and referenced, and provide one source of information to help prioritise research investment [2].

Several investigations have found that evidence syntheses, such as systematic reviews with metaanalysis, received a higher citation rate compared to other study designs [4-8]; but typically represented only $4-6 \%$ of research output $[8,9]$. It is unclear whether 'overviews of systematic reviews' (henceforth called overviews) are cited equally as highly as systematic reviews, but it stands to reason that they might considering they are a type of evidence synthesis.

The number of systematic reviews has reached epidemic proportions [10]; from none in 1987 [11]; 7 per day in 2003 [5]; 11 a day in 2010 [12]; and 22 per day in 2014 [13]. Overviews have grown in number in 
tandem with the growth in systematic reviews, and they aim to appraise and synthesise results of systematic reviews. One investigation found a five-fold increase in the publication of overviews from 2004 to 2011 [14]. The current number of overviews, and whether the trend in annual publication rate is increasing is unknown.

To advance the development of overviews, research is required to understand epidemiological aspects such as annual prevalence and factors that may influence the citation of overview publications. This paper is the first of two companion papers. In this first paper we present our evaluation of the bibliometric characteristics of overviews, and evaluate factors affecting citation rates and journal impact factor. In a second paper, we will present the assessment of whether two or more overviews overlapped in topic.

\section{Methods}

\subsection{Study design}

The design is a bibliometrics study in the knowledge synthesis field. We followed systematic review methods guidance for searching, study selection, and data extraction.

\section{$2.2 \quad$ Eligibility criteria}

We included overviews published in any language and reports were translated by one of the authors (French, Spanish, German, Mandarin), where needed. We used Google translate to translate articles in languages other than the ones listed, and when authors were not native speakers of French, Spanish, German, or Mandarin. We restricted our search to reports published from January 1, 2000 to December 2020. Given that the Cochrane Handbook chapter on overviews was first published in 2009 , we did not expect to identify overviews published prior to 2000 .

We reasoned that overviews may exist as stand-alone reports or also packaged as part of a clinical practice guideline and health technology assessment; accordingly, we developed eligibility criteria for both circumstances.

Inclusion criteria for overviews:

- Aims to primarily focus on synthesizing systematic reviews with or without meta-analyses (but the overview may also include primary studies). 
- Uses a systematic literature search found in the main body of the paper (i.e. search strategy includes text words and $\mathrm{MeSH}$ terms in at least two databases).

- Has a methods section in the main body of the paper (not just in the abstract).

- Examines the effects of health interventions or clinical treatments.

We excluded overviews that based their results solely on primary studies and methodological studies. Reports that were editorials, letters, or comments were also excluded. Overviews of risk, exposure, prevention, measurement instruments, quality indicators, diagnostic, screening or prognostic research were excluded. We excluded protocols of overviews.

Inclusion criteria for clinical practice guidelines (CPGs) and health technology assessments (HTAs):

- Uses an 'overview of review' approach to inform their recommendations (i.e. the CPG aims to primarily include, synthesise and present the results of the systematic reviews; but may also include primary studies).

\subsection{Search}

Overviews were retrieved using a validated search filter for overviews [15] from MEDLINE (Ovid), Epistemonikos and the Cochrane Database of Systematic Reviews. In an empirical methods study of the retrieval sensitivity of 6 databases, the combination of Medline and Epistemonikos retrieved $95.2 \%$ of all systematic reviews [16]. We are therefore confident that using both a validated search filter and this proven database combination will retrieve the majority of published overviews of healthcare interventions and clinical treatment in the literature.

We searched from 2000 to December 2020 (Appendix A). The Epistemonikos search was limited to the category called "Broad Syntheses" which includes overviews of systematic reviews, health technology assessments and clinical practice guidelines. Epistemonikos contains reports from electronic databases as well as the grey literature, including: PubMed, Embase, The Cumulative Index to Nursing and Allied Health Literature, PsycINFO, Literatura Latinoamericana y del Caribe en Ciencias de la Salud, Database of Abstracts of Reviews of Effects, the Campbell Collaboration's online library, the Joanna Briggs Institute (JBI) Database of Systematic Reviews and Implementation Reports, Cochrane Database of Systematic Reviews, and the Evidence for Policy and Practice Information and Co-ordinating Centre (EPPI-Centre) Evidence Library. 


\section{$2.4 \quad$ Screening and study selection}

The initial search results were imported into Excel 2010 for screening. A pilot screening of the first 19 papers was conducted in duplicate by all screeners to ensure high levels of agreement and common definitions of coding. We screened the titles and abstracts against the stated eligibility criteria first, then eligible full-text articles were reviewed for inclusion. Two independent reviewers screened reports at the title and abstract, and then again at the full text stage, then compared their results (GS, YWZ, CL, LL, PHY, $\mathrm{MD}, \mathrm{PN})$. Discrepancies were resolved by consensus, and arbitration by a third reviewer when necessary.

Categorized as 'other sources' in our flowchart (Figure 1), 237 overviews published between 2013 and 2016 were previously identified and screened $[15,17]$ (Appendix A), and added to our list of included studies.

\section{$2.5 \quad$ Data extraction}

We extracted data on overviews published between 2000 and $2018(n=541)$ to enable a feasible extraction of data. Data extraction was piloted on 20 studies by all authors independently to identify any missing variables, come to agreement on coding definitions, and refine/reword the items. Discrepancies in the piloting phase were discussed and consensus reached by two authors. Full data extraction was performed independently by one investigator (TN, AS, GS, ST, SA, UE, EKR, CL), and checked by a second reviewer (TN, AS, GS, ST, SA, UE, EKR, CL).

We extracted the medical field of the overview; year of publication; country and continent (Europe, North America, Asia, Australia, South America, Africa) of corresponding author; number of authors; journal of publication; number of included studies; search date; inclusion of meta-analysis; and funding status. We also extracted the number of total patients in the included systematic reviews. If the total number was not reported, we added up total patients across all systematic reviews from a table of characteristics. We extracted the number of times the overview had been cited per year via the Google Scholar database, and the 2-year impact factor for the top journals from Web of Science. Impact factor, as calculated by Clarivate Analytics, is a measure of the average number of times articles from a two-year time frame have been cited in a given year. We checked whether the journal was open access via the Directory of Open Access Journals (https://doaj.org/). We also extracted data on the terminology used to describe overviews in the title (overview of reviews, meta-review, review of reviews, or any related synonyms). 
The medical field categorisation used was the 10th revision of the International Statistical Classification of Diseases and Related Health Problems (ICD), a medical classification list by the World Health Organization (WHO) (https://www.who.int/standards/classifications/classification-of-diseases). Time it took to conduct the overview was not reported in the overviews. Therefore, to estimate the time for each overview's search end date to date of publication (i.e. publication lag), we calculated the difference between the initial literature search date and publication date using the month and day function in Excel 2010.

\subsection{Data analysis}

Descriptive analysis using frequencies and percentages were performed for categorical data and median and interquartile range (IQR) for continuous data. Citation density was used instead of citation count to avoid bias towards older articles. Citation density was defined as total number of citations divided by the number of years a study has been published. Citation density (the dependent variable) was positively skewed; therefore, the natural log transformation was obtained to approach a normal distribution.

Mean publication frequencies by year were calculated and presented in figures. The distribution of overviews by medical discipline and terms used to describe overviews was described. A free version of StatPlanet Plus (http://www.statsilk.com/software/statplanet) software was used to create the interactive world map to visualize the distribution of all overviews among different countries.

A multivariate regression was conducted to determine the association between predictor variables (number of authors, open versus closed journal access, number of overviews with meta-analyses, funding status, sample size, complementary and alternative therapies (CAM) topic) and two dependent variables (citation density and impact factor). Statistical significance was set at $p<.05$. Statistical analyses were performed using Stata version 13 (StataCorp LP) using two commands manova and mvreg.

\section{Results}

\subsection{Search results}

From a total of 16,610 records retrieved from the MEDLINE, Cochrane Database of Systematic Reviews, and Epistemonikos databases, and 237 records retrieved from other sources (Figure 1), 2410 duplicates were removed. Of the remaining 14,437 records, 11,481 were excluded at the title/abstract stage, and 1738 were excluded at the full text stage. A total of 1218 overviews were included that met our eligibility criteria, and of these, 541 overviews published between 2000 and 2018 (44\%) were data extracted (data 
set can be accessed at https://osf.io/38bkg/). Many studies did not have a methods section, did not conduct a systematic search, and did not search for and include systematic reviews (Figure 1).

\subsection{Prevalence of overviews}

We identified 1218 overviews, CPGs or HTAs published between 2000 and 2020. Between the years of 2000 and 2018, there has been an increase in the publication of overviews (Figure 2). The majority of overviews (886/1218 [73\%]) were published between a 5-year period, from 2016-2020. Approximately three-quarters of the overviews (1117/1218 [92\%]) were reported during the most recent decade (20102020). An 8-fold increase in the number of overviews was found between $2009(n=25)$ and $2020(n=332)$. We identified 332 overviews published in the three databases in 2020 , which is approximately equivalent to 1 overview published per day.

\subsection{Characteristics of included overviews published between 2000 and $2018(n=541)$}

Overviews published between 2000 and 2018 were extracted and analysed $(n=541)$. The most frequent subject of the overviews (92/541 [17\%]) was ICD-10 classification "factors influencing health status and contact with health services." Another 62/541 (11.5\%) focused on diseases of the musculoskeletal system and connective tissue, 56/541 (10.4\%) were about mental and behavioural disorders, 42/541 (7.8\%) were on diseases of the circulatory system, and 34/541 (6.3\%) were focused on neoplasms. A little under half of the overviews focused on 15 other conditions in the ICD-10 medical classification (Table 1).

Table 1: Characteristics of overviews published between 2000 and $2018(n=541)$ 


\section{Characteristic}

ICD-10 medical classification

\section{Category}

Frequency

(\%)

Factors influencing health status and contact with health services

$92(17 \%)$

Diseases of the musculoskeletal system and connective tissue

Mental and behavioural disorders

Diseases of the circulatory system

Neoplasms

Diseases of the nervous system

Diseases of the respiratory system

Other (13 more ICD-10 classifications with under 26 overviews per topic)
Complementary and Alternative Medicine topic

Number of authors per overview

\begin{tabular}{ll}
\hline 1 & $22(4 \%)$ \\
\hline $2-3$ & $163(30 \%)$ \\
\hline $4-6$ & $219(41 \%)$ \\
\hline $7-10$ & $88(16 \%)$ \\
\hline $11-20$ & $36(7 \%)$ \\
$>21$ & $8(2 \%)$ \\
\hline
\end{tabular}

Total number of countries of corresponding authors 42

Country of corresponding author

\begin{tabular}{l} 
United Kingdom \\
United States \\
\hline Canada \\
Australia \\
\hline China \\
\hline Germany \\
\hline The Netherlands \\
\hline Brazil \\
\hline $\begin{array}{l}\text { Other (34 more countries with under } 10 \\
\text { overviews) }\end{array}$
\end{tabular}




\begin{tabular}{|c|c|c|}
\hline & North America & $125(23 \%)$ \\
\hline & Asia & $61(11 \%)$ \\
\hline & Australia/NZ & $46(9 \%)$ \\
\hline & South America & $37(7 \%)$ \\
\hline & Africa & $5(1 \%)$ \\
\hline \multirow[t]{2}{*}{ Presence of meta-analysis } & No & $465(86 \%)$ \\
\hline & Yes & $76(14 \%)$ \\
\hline \multirow[t]{2}{*}{ Funding } & Not reported & $269(50 \%)$ \\
\hline & Reported & $272(50 \%)$ \\
\hline \multirow[t]{2}{*}{ Number of included SRs (sample size) } & Mean (SD) & $24.3(32)$ \\
\hline & Median (IQR) & $14(7-27)$ \\
\hline \multirow[t]{2}{*}{$\begin{array}{l}\text { Publication lag (time from search end } \\
\text { date to date of publication) }\end{array}$} & Mean (SD) & $\begin{array}{l}1.6 \text { years } \\
(2.3)\end{array}$ \\
\hline & Median (IQR) & $\begin{array}{l}1 \text { year }(1- \\
2)\end{array}$ \\
\hline \multirow[t]{2}{*}{ Number of included patients } & Mean (SD) & $\begin{array}{l}109.7 \\
(113.4)\end{array}$ \\
\hline & Median (IQR) & $75(1-209)$ \\
\hline \multicolumn{2}{|c|}{ Overview that directly informed a clinical practice guideline } & $17(3 \%)$ \\
\hline \multicolumn{2}{|c|}{ Clinical practice guideline (using an overview of reviews for evidence synthesis) } & $30(6 \%)$ \\
\hline \multicolumn{2}{|l|}{ Cochrane overviews } & $44(8 \%)$ \\
\hline
\end{tabular}

SRs: systematic reviews; SD: standard deviation; ICD: 10th revision of the International Statistical Classification of Diseases and Related Health Problems; IQR: interquartile range

Several authors working on one overview was common; the average authorship per overview was 5.3, with number of authors ranging from 1 to 24 . Overviews most frequently had 4 to 6 authors (219 [41\%]) on the team. Corresponding authors were most frequently from the United Kingdom (140/541 [26\%]), the United States (65/541 [12\%]), or Canada (53/541 [10\%]) (Figure 3). The largest proportion of overviews (267/541 [49\%]) had a corresponding author from Europe while the smallest proportion of overviews (5/541 [1\%]) had a corresponding author from Africa. The median number of included systematic reviews was 14 (interquartile range [IQR] 7-27), and the mean number of patients was 110 . A majority (465/541 [86\%]) did not conduct a meta-analysis, and half $(272 / 541$ [50\%]) reported funding 
sources. The mean time from search to publication was 1.6 years.

\subsection{Journals, impact factor, and citation density of overviews published between 2000 and 2018 ( $n=541)$}

Authors of 541 overviews published in 307 journals between 2000 and 2018 (Table 2). The majority of overviews (369/528 [70\%]) published in journals with impact factors between 0.05 and 3.97. Thirty percent of overviews (159/528) were published in journals with impact factors of 4 or greater. Twenty overviews published in journals with impact factors between 17 and 59 (e.g. The Lancet, BMJ, JAMA). The Cochrane Database of Systematic Reviews published the largest number of overviews (44/538 [8\%]), followed by PLOS ONE (16/538 [3\%]), the Sao Paulo Medical Journal (12/538 [2\%]), and BMJ Clinical Evidence (10/538 [1.9\%]). About half (227/538 [42\%]) of the overview published in unique journals (i.e. only one overview published in that journal). 130 overviews (24\%) published in open access journals or chose an open access option when publishing.

Table 2: Journals, impact factor, and citation density of 541 overviews published between 2000 and 2018 


\begin{tabular}{|c|c|c|}
\hline Journal characteristic & Category & Frequency (\%) \\
\hline Number of journals & & 307 \\
\hline \multirow[t]{5}{*}{ Number of overviews per journal } & 1 & 227 \\
\hline & $2-4$ & 56 \\
\hline & $5-10$ & 18 \\
\hline & $11-16$ & 2 \\
\hline & 44 & 1 \\
\hline \multirow[t]{8}{*}{ Journals } & Cochrane Database of Systematic Reviews & $44(8 \%)$ \\
\hline & PLOS ONE & $16(3 \%)$ \\
\hline & Sao Paulo Medical Journal & $12(2 \%)$ \\
\hline & BMJ Clinical Evidence & $10(1.8 \%)$ \\
\hline & BMJ Open & $7(1.3 \%)$ \\
\hline & British Journal of Sports Medicine & $7(1.3 \%)$ \\
\hline & Maturitas & $7(1.3 \%)$ \\
\hline & Other (<299 journals) & $439(81 \%)$ \\
\hline \multirow[t]{7}{*}{ Journal impact factor } & $0.01-1.99$ & $153(29 \%)$ \\
\hline & $2.0-3.97$ & $216(41 \%)$ \\
\hline & $4.0-6.96$ & $61(11 \%)$ \\
\hline & $7.0-12.79$ & $79(14 \%)$ \\
\hline & $13.6-59.1$ & $23(4 \%)$ \\
\hline & Median (IQR) & $2.8(1.9-4.6)$ \\
\hline & Mean (SD) & $4.40(5.44)$ \\
\hline Open access journals & & $130(24 \%)$ \\
\hline \multirow[t]{6}{*}{ Citation Density } & $0.0-1.0$ & 78 (14.4\%) \\
\hline & $1.1-3.0$ & 79 (14.6\%) \\
\hline & $3.1-5.0$ & $62(11.5 \%)$ \\
\hline & $5.1-10.0$ & $98(18.1 \%)$ \\
\hline & $10.3-20.0$ & $117(21.6 \%)$ \\
\hline & $20.1-40.4$ & 62 (11.5\%) \\
\hline
\end{tabular}


SRs: systematic reviews; SD: standard deviation; IQR: interquartile range

We found an average citation rate of 90 (SD \pm 219.7 ) over 9 years, or 10 citations per overview per year. When examining overviews published in the top 50 journals by impact factors ( $\geq 7.86$ ), we found that 50 overviews $(9 \% ; 50 / 537)$ received $18 \%$ of citations (range $4-1250)$. Nine overviews remained un-cited. A total of 108 out of 537 overviews (20\%) were cited 100 times or more. An article having 100 or more citations is regarded as a 'classic' [18]. The topics of the six overviews with citations counts of $>1000$ included two on diseases of the musculoskeletal system and connective tissue, two on factors influencing health status and contact with health services, and one each on pregnancy, childbirth and the puerperium; and mental and behavioural disorders.

\subsection{Nomenclature of 541 overviews published between 2000 and 2018}

Overall, $14 \%(73 / 541)$ of the overviews did not use any terms or synonyms to identify the study as an overview (Appendix B). Of the 541 overviews, the majority used the term overview (196/541 [36\%]) and 'systematic review of reviews' (74/541 [14\%]) or a close derivative in the title (Appendix B). Synonyms and other terms were used less frequently; 42 overviews (8\%) used the term umbrella review, and $19(4 \%)$ used the term meta-review (3\%) in the title. The term 'systematic review' was used in 33/541 (7\%) to mean an overview.

A word cloud was created to visualize the frequency of words and phrases used, with the sizes of words and phrases representing the frequency (Figure 4).

\subsection{Multivariate regression}

Multiple regression analyses were conducted to examine the relationship between citation density and impact factor with various potential predictors. Controlling for the other variables in the model, citation density and impact factor are positively and significantly correlated with the number of authors, sample size, funding reported, and open access articles (Appendix C). This indicates that overviews with a high number of citations and high journal impact factors tend to have a higher number of authors, larger sample sizes, be open access and report sources of funding. The multiple regression model with impact factor, and four predictors, produced $\mathrm{R}^{2}=.119, \mathrm{~F}(5,514)=17.18, \mathrm{p}<.001$, and citation density and four predictors produced $\mathrm{R}^{2}=.126, \mathrm{~F}(5,514)=18.31, \mathrm{p}<.001$. Overviews on topics related to CAM and overviews using a meta-analysis were not associated with citation density and impact factor, and were therefore not included in the model.

\section{Discussion}




\subsection{Summary of results}

To our knowledge, this is the first study to examine the overview annual publication prevalence, predictors of citation rates and journal impact factors. This bibliometrics study provides a baseline of publication trends and impact of overviews, and the list of overviews identified can be used in future methods studies. The number of overviews has increased over the last 20 years, with $73 \%$ of the overviews published in the most recent 5-year period (2016-2020). In 2020, one overview was published per day. The majority of overviews had 4 to 6 authors on the team, and took on average 1.6 years to produce from search date to publication. Most overviews were published on the medical categories of health systems and services, musculoskeletal system, and mental disorders. The journals that publish the most overviews were the Cochrane Database of Systematic Reviews, PLOS ONE, and the Sao Paulo Medical Journal, but each with $<10 \%$ of the published overview sample.

\subsection{Prevalence of overviews}

We found an 8-fold increase in the number of overviews between $2009(n=25)$ and $2020(n=332)$. An annual increasing prevalence of overviews has been found by other authors. One study found that the number of overviews increased from 1 in 2000 to 14 in 2010 [19] and another found a five-fold increase in the publication of overviews from 2004 to 2011 [14]. The factors driving the increase in overviews are explained by the exponential increase in the number of systematic reviews being published, the publication of guidance on how to conduct an overview of reviews by Cochrane as of 2009, the subsequent publication of the first Cochrane overview in the same year.

\subsection{Higher citation rate of overviews}

We found that the average citation rate for overviews was 90 (SD \pm 219.7 ) over 9 years, or 10 citations per overview per year. Several studies have found that systematic reviews and meta-analyses have higher citation counts than RCTs or observational studies [4, 20-23]. Mean citation counts for systematic reviews and meta-analyses ranged from 0.74 [4], 5.1 [22] to 6.6 per year [23]. Our study found a significantly higher mean citation count of 10 overviews per year than these studies on reviews. The mean impact factor of the journals the overviews publish is was 4.4. These data indicate that, overall, overviews perform above average for the journals in which they publish, and therefore may contribute to increasing the two-year impact factors of the journals in which they are published.

\subsection{Terminology}


Currently identifying and searching for overviews is problematic because there is no agreed nomenclature, and no standardised definition. One of the first overviews was published in 1992 [24]; however, it only became recognized as such after 2009, when Cochrane published guidance for overviews, and its first overview in the Cochrane library.

We found that only one third of our sample used the term was overview in the title, $12 \%$ used umbrella review or meta-review, and $14 \%$ used systematic review of reviews, or a close derivative. Of concern is that $7 \%$ used the term 'systematic review' to mean an overview of reviews. Inconsistent nomenclature may have affected the performance of our search filter used in this study. Overviews entitled 'systematic reviews' would be miscoded into MEDLINE's Publication Type (PT) for systematic reviews, leading to a compounding problem. Overviews should have their own Publication Type (PT) term in MEDLINE to help differentiate systematic reviews from overviews [15].

A consistent nomenclature for overviews would help in the identification of overviews. We recommend that authors of overviews use the term overview of systematic reviews in the title to help users search for and identify relevant reports.

\subsection{Predictors of citation and impact factor}

In our large sample, overviews with a high number of citations and high journal impact factors had group authorship, large sample sizes, were open access, and reported sources of funding. Several studies of citation counts and impact factors in the health field identified predictors of citation rates similar to our own, namely number of authors, sample size, funding status, and open access $[20,21,23,25,26]$.

\subsection{Strengths and limitations}

The main strength of this study is that it is the first to look at predictors of citations and journal impact factor specific to overviews published over a wide range of subject areas and journals, and it included a number of characteristics of both the article itself and the journal in which it appeared. Other strengths include comprehensiveness of years searched, the large number of extracted articles $(n=541)$, and our strict eligibility criteria for overviews. We also search a combination of databases (Medline, Epistemonikos and Cochrane) with a high sensitivity (over 95\%) in retrieving overviews in all medical fields [16]. 
A limitation to our study is that we may not have captured the actual number of overviews published as we only searched three databases and did not search the grey literature. Furthermore, we used a verification system for data extraction rather than independent extraction, which may not be as robust as what Cochrane standards are for Cochrane reviews. Less rigorously developed overviews, such as those only searching one database, were not included. Also, our selected overviews were limited to those pertaining to therapeutic, clinical and health intervention topics. In addition, the 541 analyzed do not represent the whole sample of 1218 overviews, this limiting generalizability of our findings.

Our ability to predict the variance of annual citation rates was weak (13\%); however, it is consistent with other similar studies. One study predicted $14 \%$ of the variance of annual citation rates using emergency medicine studies and 12 possible predictor variables [27], and another study predicted $20 \%$ using articles from three high impact journals [25].

\subsection{Future research}

The research provides a baseline of publication trends and impact of overviews for future research. Future research could also explore whether the quality of conduct of overviews predicted citation density. An examination of the top 100 cited overviews compared to those least cited would inform authors about what factors contribute to citation rate. In addition, future investigations should explore social media impact metrics (e.g. altmetric score) and other article metrics, and composition of overview author networks. Finally, this compilation of 1218 overviews represents a bibliographic database of all overviews published in healthcare interventions or clinical treatments from 2000 to 2020 and can be used by methodologists interested in studying this review type.

\subsection{Conclusions}

This 20-year bibliometric study is the first of its kind to identify and characterize overviews across 307 journals indexed in three databases. The 8-fold increase in the number of overviews from the publication of the Cochrane guidance for overviews in 2009 to 2020 illustrates the increasing popularity and demand for overviews. This demand is due potentially to the need to synthesise the results of multiple reviews on topics of broad scope, and of relevance to policy and practice.

Many academics, practitioners, and policy makers have failed to see the impact and utility of overviews, and their potential to inform clinical practice, policy and guideline development. Citation analysis and impact factor metrics can help these knowledge users to quantify, compare, and communicate the 
influence of overviews of reviews. Our study found a mean citation count of 10 overviews per year, published in journals with a mean impact factor of 4.4. These data indicate that, overall, overviews perform above average for the journals in which they publish, and therefore may contribute to increasing their two-year impact factors. We also found that highly cited overviews in journals with high impact factors are associated with group authorship, large sample sizes, open access publishing, and reported sources of funding. The study provides a baseline of annual publication trends and bibliometric impact of overviews for future research.

\section{Highlights}

- Overviews synthesise the results of multiple systematic reviews.

- Many academics, practitioners, and policy makers have failed to see the impact and utility of overviews, and their potential to inform clinical practice, policy and guideline development.

- Citation analysis and impact factor metrics can quantify, compare, and communicate the influence of overviews of reviews.

- Our study reports the results of an empirical study investigating the prevalence, characteristics, number of citations of 'overviews of reviews' indexed in MEDLINE®, Epistemonikos, and the Cochrane Database of Systematic Reviews.

- No bibliometric study has been published on overviews of reviews.

- Our study findings show the growing prevalence of overviews, and an 8-fold increase in the number of overviews published from 2009 to 2020 , representing one published overview a day.

- We found a high mean citation count of 10 overviews per year, published in journals with a mean impact factor of 4.4. These data indicate that, overall, overviews perform above average for the journals in which they publish.

\section{Declarations}

\section{Ethics approval and consent to participate}

N/A

\section{Transparency declaration}

The lead author affirms that this manuscript is an honest, accurate, and transparent account of the study being reported; that no important aspects of the study have been omitted; and that any discrepancies from the study as planned (and, if relevant, registered) have been explained. 


\section{Data sharing and availability of data and materials}

The full dataset is available on https://osf.io/38bkg

\section{Competing interests}

The authors have declared no competing interest.

\section{Funding}

This work did not receive any funding.

\section{Dissemination declaration}

\section{The results were disseminated in conference and university workshops, social media, and email lists.}

\section{Authors' contributions}

$\mathrm{CL}$ conceived of the study design, wrote the study protocol, analyzed the data, wrote and revised the final manuscript. GS, YWZ, CL, LL, PHY, MD, PN screened citations and full-text articles. TN, AS, GS, ST, SA, UE, EKR abstracted and verified data. CL, TN, AC, GS, SS analysed the data. CL, AC, AS, BJS interpreted results, and edited the manuscript. TN, AC, GS, AS, ST, SS, SA, YWZ, LL, PHY, MD, PN, UE, BJS, EKR, and JMW edited and approved of the final manuscript,

\section{Acknowledgements}

No acknowledgements.

\section{References}

1. Retrouvey, $\mathrm{H}_{\text {., }}$ et al., Cross-sectional analysis of bibliometrics and altmetrics: comparing the impact of qualitative and quantitative articles in the British Medical Journal. BMJ Open, 2020. 10(10): p. e040950.

2. Brandt, J.S., et al., A Bibliometric Analysis of Top-Cited Journal Articles in Obstetrics and Gynecology. JAMA Network Open, 2019. 2(12): p. e1918007-e1918007. 
3. Trueger, N.S., Y. Yilmaz, and T.M. Chan, Leveraging Tweets, Citations, and Social Networks to Improve Bibliometrics. JAMA Network Open, 2020. 3(7): p. e2010911-e2010911.

4. Allareddy, V., et al., Association between study design and citation counts of articles published in the American Journal of Orthodontics and Dentofacial Orthopedics and Angle Orthodontist. Orthodontics (Chic.), 2012. 13(1): p. 184-91.

5. Montori, V.M., et al., Systematic reviews: a cross-sectional study of location and citation counts. BMC Med, 2003. 1: p. 2.

6. Patsopoulos, N.A., A.A. Analatos, and J.P. loannidis, Relative citation impact of various study designs in the health sciences. Jama, 2005. 293(19): p. 2362-6.

7. Winnik, S., et al., From abstract to impact in cardiovascular research: factors predicting publication and citation. Eur Heart J, 2012. 33(24): p. 3034-45.

8. Willis, D.L., et al., Predictors of citations in the urological literature. BJU international, 2011. 107(12): p. 1876-1880.

9. Wolf, D.M. and P.A. Williamson, Impact factor and study design: the Academic Value of Published Research (AVaRes) score. The Annals of The Royal College of Surgeons of England, 2009. 91(1): p. 71-73.

10. Ioannidis, J.P.A., The Mass Production of Redundant, Misleading, and Conflicted Systematic Reviews and Meta-analyses. The Milbank quarterly, 2016. 94(3): p. 485-514.

11. Mulrow, C.D., The medical review article: state of the science. Ann Intern Med, 1987. 106(3): p. 485-8.

12. Bastian, H., P. Glasziou, and I. Chalmers, Seventy-five trials and eleven systematic reviews a day: how will we ever keep up? PLoS Med, 2010. 7(9): p. e1000326.

13. Page, M.J., et al., Epidemiology and Reporting Characteristics of Systematic Reviews of Biomedical Research: A Cross-Sectional Study. PLoS Med, 2016. 13(5): p. e1002028.

14. Pieper, D., et al., Overviews of reviews often have limited rigor: a systematic review. J Clin Epidemiol, 2012. 65(12): p. 1267-73.

15. Lunny, C., J.E. McKenzie, and S. McDonald, Retrieval of overviews of systematic reviews in MEDLINE was improved by the development of an objectively derived and validated search strategy. J Clin Epidemiol, 2016. 74: p. 107-18.

16. Goossen, K., et al., Database combinations to retrieve systematic reviews in overviews of reviews: a methodological study. BMC medical research methodology, 2020. 20(1): p. 138-138. 
17. Lunny, C., et al., Overviews of reviews incompletely report methods for handling overlapping, discordant, and problematic data. Journal of Clinical Epidemiology, 2020. 118: p. 69-85.

18. Andersen, J., J. Belmont, and C.T. Cho, Journal impact factor in the era of expanding literature. Journal of microbiology, immunology, and infection= Wei mian yu gan ran za zhi, 2006. 39(6): p. 436443.

19. Hartling, L., et al., A descriptive analysis of overviews of reviews published between 2000 and 2011. PLoS One, 2012. 7(11): p. e49667.

20. Lokker, C., et al., Prediction of citation counts for clinical articles at two years using data available within three weeks of publication: retrospective cohort study. BMJ, 2008. 336(7645): p. 655-657.

21. Tahamtan, I., A.S. Afshar, and K. Ahamdzadeh, Factors affecting number of citations: a comprehensive review of the literature. Scientometrics, 2016. 107(3): p. 1195-1225.

22. Bhandari, M., et al., Factors associated with citation rates in the orthopedic literature. Canadian journal of surgery. Journal canadien de chirurgie, 2007. 50(2): p. 119-123.

23. Royle, P., et al., Bibliometrics of systematic reviews: analysis of citation rates and journal impact factors. Systematic Reviews, 2013. 2(1): p. 74.

24. Antman, E.M., et al., A comparison of results of meta-analyses of randomized control trials and recommendations of clinical experts. Treatments for myocardial infarction. Jama, 1992. 268(2): p. 240-8.

25. Kulkarni, A.V., J.W. Busse, and I. Shams, Characteristics associated with citation rate of the medical literature. PLoS One, 2007. 2(5): p. e403.

26. Davis, P.M., et al., Open access publishing, article downloads, and citations: randomised controlled trial. BMJ, 2008. 337: p. a568.

27. Callaham, M., R.L. Wears, and E. Weber, Journal prestige, publication bias, and other characteristics associated with citation of published studies in peer-reviewed journals. Jama, 2002. 287(21): p. 2847-50.

\section{Figures}


Records identified through databases

- $\operatorname{MEDLINE}$ (Ovid) $(\mathrm{n}=11,839)$

- Epistemonikos ( $n=4646$ )

- Cochrane Database of Systematic Reviews ( $n=125$ )

$\longrightarrow$\begin{tabular}{l}
$\begin{array}{l}\text { Records identified through } \\
\text { other sources } \\
\text { Overviews identified from } 2 \\
\text { previous studies }(\mathrm{n}=237)\end{array}$ \\
\hline
\end{tabular}

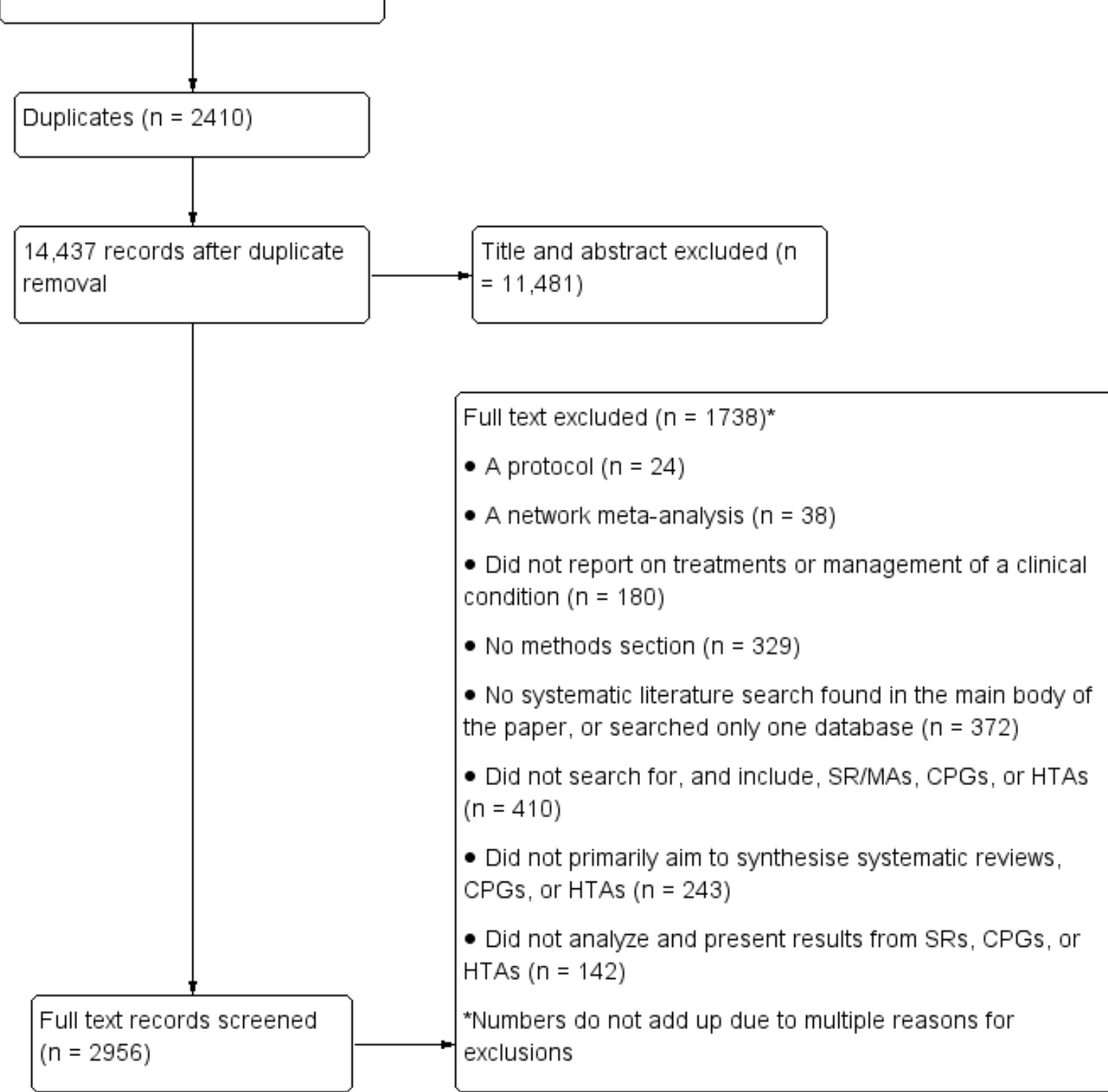

1218 overviews of healthcare interventions included

\section{Figure 1}

PRISMA flowchart of study selection 


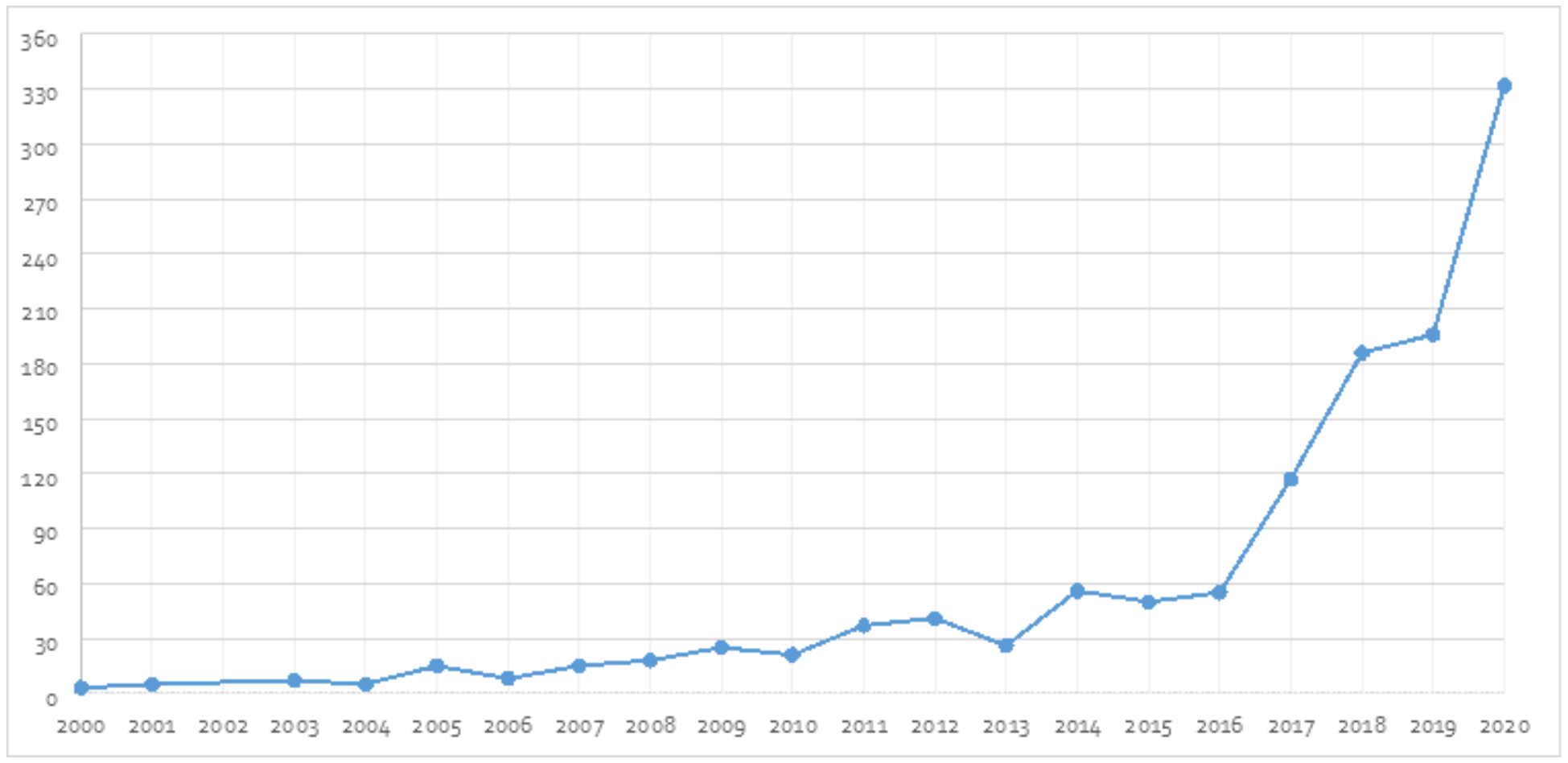

Figure 2

Frequency of overviews published per year (2000 to 2020)

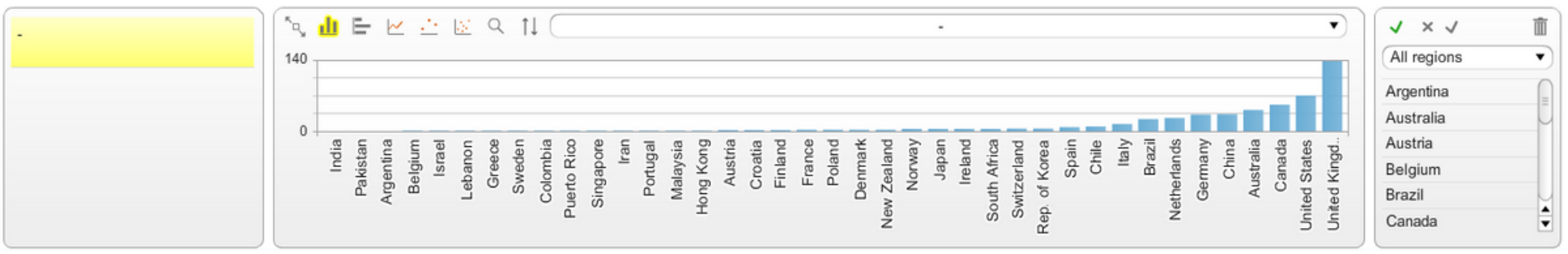

$\square>23$
$16-23$
$9-16$
$2-9$
$\square<2$
$\square$ No data

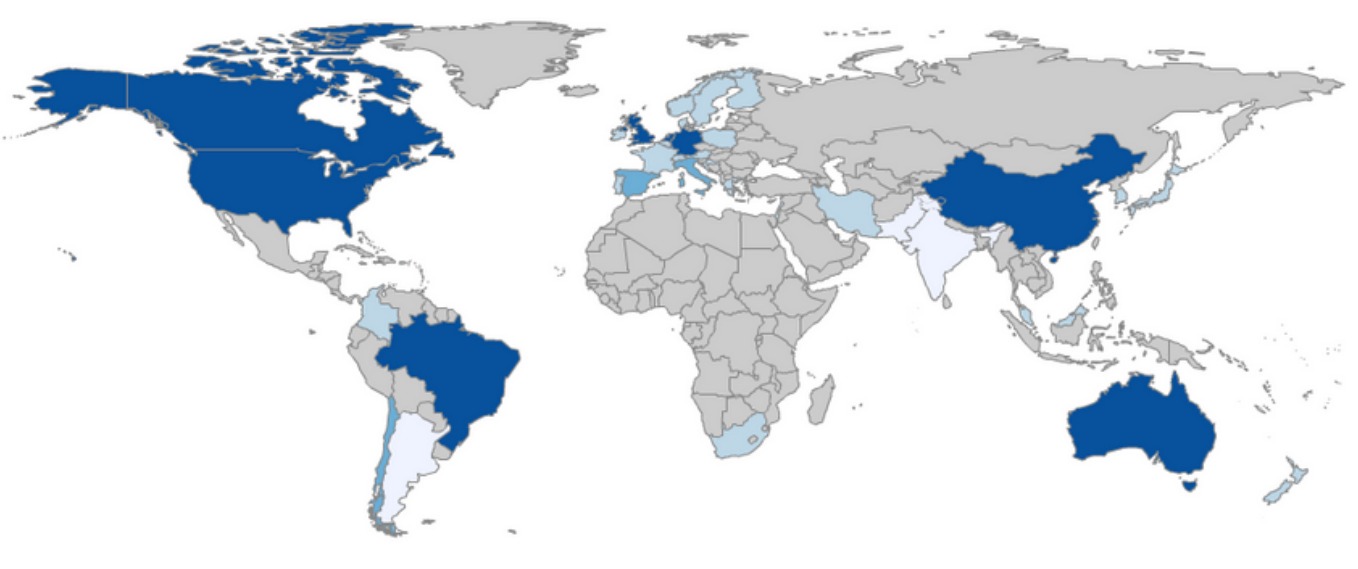

\section{Figure 3}

Map of country of corresponding authors of overviews published between 2000 and $2018(n=541)$ 


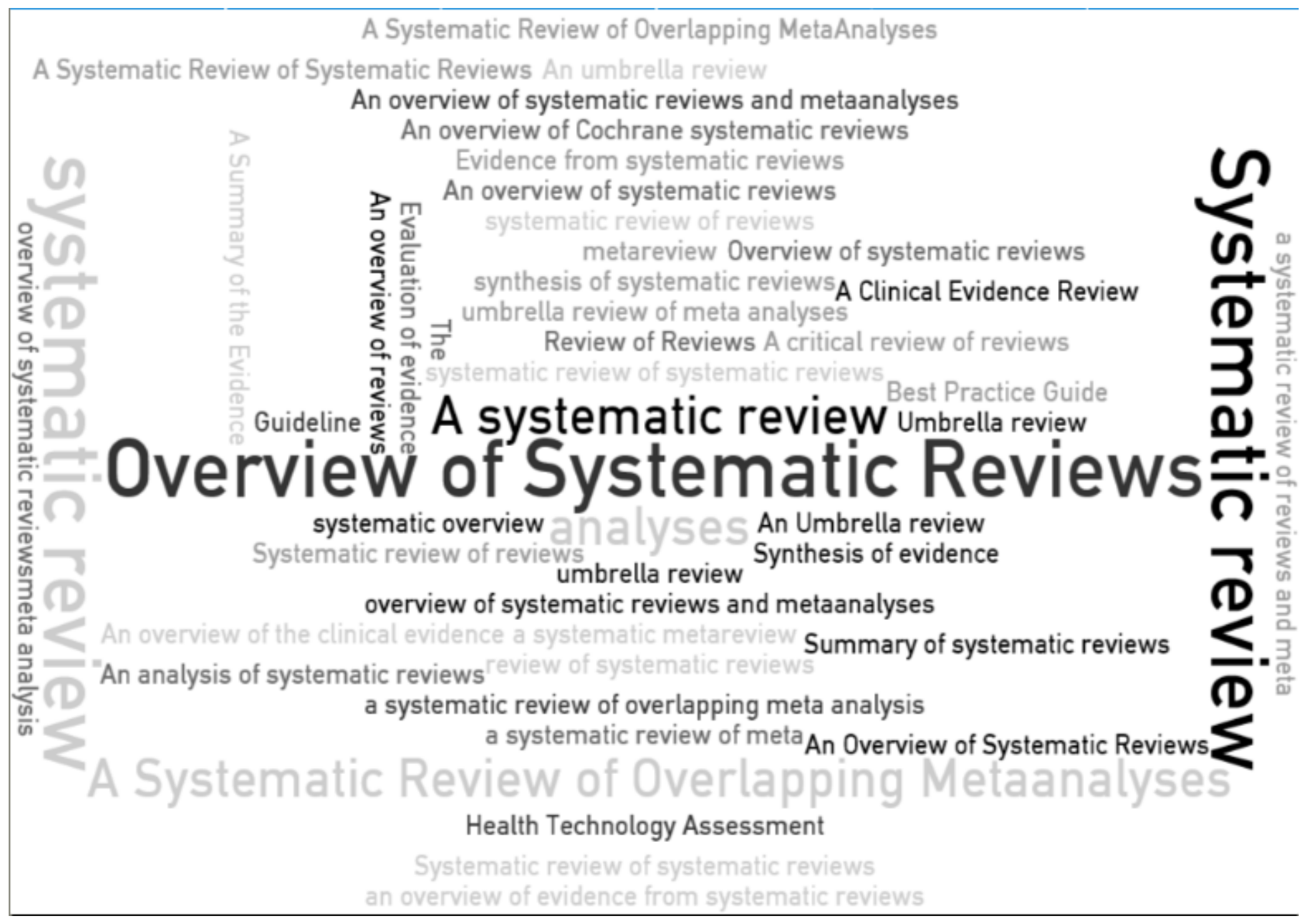

Figure 4

Word cloud of terms in titles describing 541 overviews published between 2000 and 2018

\section{Supplementary Files}

This is a list of supplementary files associated with this preprint. Click to download.

- 20210210SupplfileBibliographicstudy.docx 